\title{
A case of cholangiolocellular carcinoma in which intrahepatic cholangiocarcinoma was confirmed from appearance to development
}

\author{
Takashi Orii $\cdot$ Yukihiko Karasawa $\cdot$ Yusuke Miyagawa \\ Kenji Sano
}

Received: 11 July 2012/ Accepted: 1 October 2012/Published online: 29 October 2012

(C) The Japan Society of Clinical Oncology 2012

\begin{abstract}
We describe the case of a 68-year-old asymptomatic woman with cholangiolocellular carcinoma (CoCC) combined with intrahepatic cholangiocarcinoma (ICC). She was diagnosed with hepatic hemangioma and followed up for approximately three years. Because the hepatic tumor increased in size and was clinically diagnosed as ICC without metastasis, a hepatectomy was performed. Histopathological examination confirmed that the tumor was CoCC surrounded by a region of ICC. Analysis of the computed tomography images from the two years before the operation confirmed that the ICC arose from a part of the preexisting CoCC and increased over a short period of time. Three months after the operation, cancer recurred in the liver and regional lymph nodes; therefore, we performed chemotherapy for one year and four months, followed by hepatectomy and dissection of the lymph nodes. Microscopic findings showed that the hepatic recurrence was $\mathrm{CoCC}$, and that all of the malignant lymph nodes were metastases from ICC. This is a suggestive and valuable case that informs us of the transformation process from $\mathrm{CoCC}$ to ICC; it indicates that ICC differentiated from CoCC and adopts a different manner of metastasis from the original "mother" CoCC.
\end{abstract}

T. Orii · Y. Karasawa · Y. Miyagawa

Department of Surgery, Showa Inan General Hospital,

3230 Akaho, Komagane 399-4191, Japan

T. Orii $(\bowtie)$

Department of Surgery, Showa Inan General Hospital,

230 Akaho, Komagane 981-8501, Japan

e-mail: t.orii@sihp.jp

K. Sano

Department of Laboratory Medicine, Shinshu University School

of Medicine, Matsumoto, Japan
Keywords Cholangiolocellular carcinoma - Intrahepatic cholangiocarcinoma $\cdot$ Epithelial membrane antigen

\section{Introduction}

Cholangiolocellular carcinoma (CoCC) is believed to be derived from hepatic progenitor cells, which have the potential to differentiate into either hepatocytes or cholangiocytes $[1,2]$, so CoCC has the ability to progress to either hepatocellular carcinoma (HCC) or intrahepatic cholangiocarcinoma (ICC). Although we found some articles that report the coexistence of CoCC with $\mathrm{HCC}$ or ICC, no report has confirmed the progress of ICC or HCC from CoCC.

Herein, we present the case of a woman who underwent hepatic resection with a diagnosis of ICC after a long observation period of approximately three years with hepatic hemangioma. Histopathological examination showed that the tumor was CoCC coexisting with ICC. We report an interesting case in which we observed the process of differentiation of CoCC into ICC by retrospective analyses of computed tomography (CT) images.

\section{Case report}

The gastroenterological division of our hospital followed up the patient, an asymptomatic 68-year-old woman, for chronic hepatitis B and hepatic hemangioma from May 2005. On November 28, 2008, computed tomography (CT) showed that the tumor, which had been diagnosed as hepatic hemangioma, was suspected to be malignant due to enlargement and a change in the contrast pattern of the tumor. She therefore visited the surgical division to undergo 
hepatic surgery for the tumor. She did not have any other illness, and her general condition was satisfactory.

Figure 1 shows the changes in the CT findings, which were taken on four occasions from November 2006 to November 2008. The plain CT showed the tumor as a low-density area that was gradually increasing in segment 8 of the liver. The tumor size increased as time progressed, particularly in the left dorsal region (Fig. 1c-f). Finally, it was confirmed as a $42 \mathrm{~mm} \times 37 \mathrm{~mm}$ dumbbell-shaped tumor (Fig. 1f). The ventral part of the tumor was described as a high-density area with a few lowdensity spots in the early phase, followed by a slightly diminishing concentration of enhancement. In contrast, the dorsal part of the tumor presented with peripheral enhancement in the early phase, while in the delayed phase, the area of the enhancement spread toward the center (Fig. 1a, b).

On the T1-weighted image (T1WI) of magnetic resonance imaging (MRI), the tumor was a well-demarcated homogeneous lesion of low signal intensity (Fig. 2a). On the T2-weighted image (T2WI), the tumor was confirmed as comprising two different signal intensities: a high-intensity signal in the right ventral portion and a high-intensity signal with a large central low-intensity signal in the dorsal portion (Fig. 2b). In the dynamic study of T1WI (Fig. 2c-g), the ventral high-intensity portion had marked peripheral enhancement in the early phase, which then diminished to the same or slightly lower intensity as the surrounding liver. In contrast, the dorsal low-intensity portion had peripheral enhancement in the early phase, very slowly followed by central spotty enhancement.

In laboratory examinations, tests of liver and kidney function were within normal limits; however, the indocyanine green 15-min retention (ICGR15) test was $14.5 \%$, which indicated mild liver function insufficiency. Serological tests for hepatitis B surface antigen (EIA) were positive, but the HBV-DNA titer (TMA) was below $3.7 \mathrm{log}$ copies $/ \mathrm{mL}$. Elevation of serum tumor markers such as carcinoembryonic antigen (CEA), carbohydrate antigen 19-9 (CA19-9), alpha-fetoprotein (AFP), protein induced by vitamin $\mathrm{K}$ absence or antagonist-2 (PIVKA-2), and DUPAN-2 was not detected.

The tumor was diagnosed as ICC, which appeared in the same place as the hepatic hemangioma that was followed up for several years. On December 24, 2008, she underwent resection of segment 8 of the liver without dissection
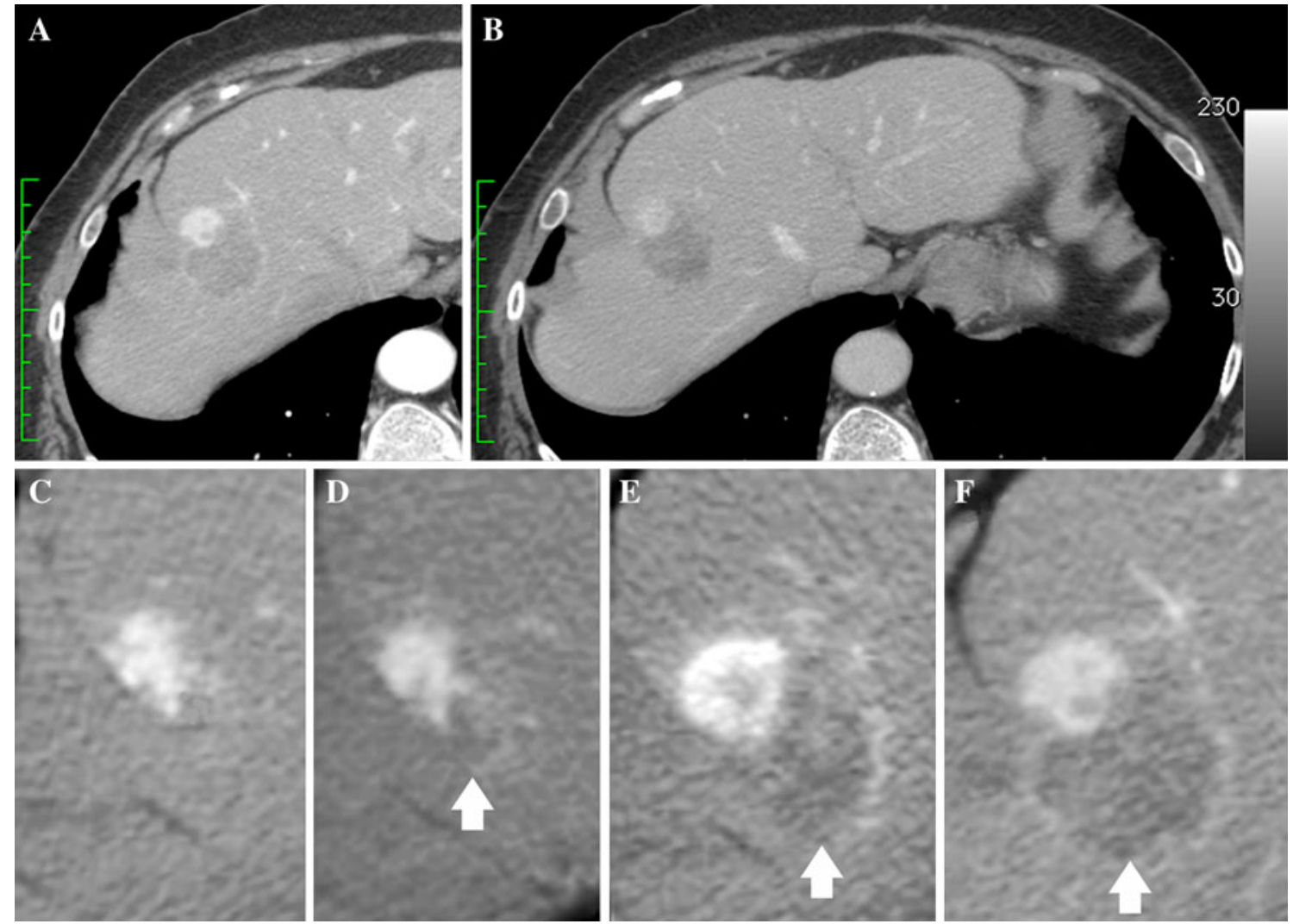

Fig. 1 Computed tomography. Early phase (a) and delayed phase (b) of dynamic contrast-enhanced images at the first operation. The changes in the images in the early phase are shown from $\mathbf{c}$ to $\mathbf{f}$. These photos were taken on c November 9, 2006, d June 5, 2007, e June 4, 2008, and $\mathbf{f}$ November 28, 2008 (just before the first operation). $\mathbf{f}$ is a higher magnification of $\mathbf{a}$. The white arrow indicates the area of ICC 

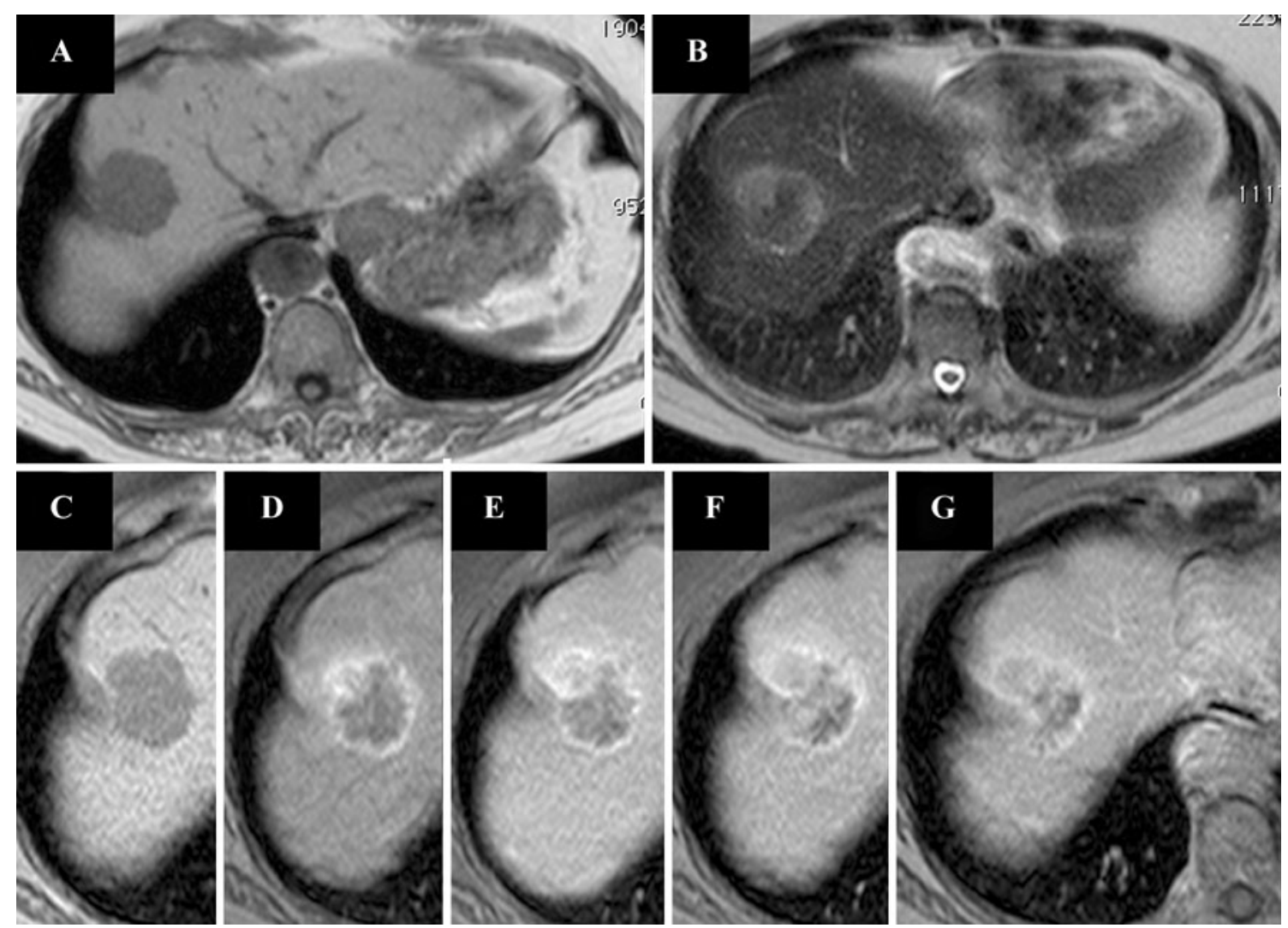

Fig. 2 Magnetic resonance imaging at the first operation: a T1WI, b T2WI. The photos $\mathbf{c}-\mathbf{g}$ are the images from a dynamic study with Gd-EOBDTPA

of regional lymph nodes due to no apparent swelling in the preoperative examination.

The gross findings for the $4.0 \times 3.5 \mathrm{~cm}$ tumor were: whitish/light yellowish in color, solid, nonencapsulated, with an irregular margin. Microscopically, in the portion of the CoCC shown in the mapping photo (Fig. 3), atypical epithelial cells proliferated in tubular structures with abundant fibrous stroma (Fig. 4a), and strongly expressed cytokeratin (CK) 7 and epithelial membrane antigen (EMA) on the luminal surface (Fig. 5a). In contrast, in the ICC portion, which surrounded the CoCC portion, the tumor cells, which formed an acinar, trabecular, or glandular pattern (Fig. 4b), were positive for CK7 and EMA in the cytoplasm (Fig. 5b). On the basis of these findings, the tumor was diagnosed as CoCC coexisting with ICC.

Three months after the operation, CT showed the appearance of recurrent lesions in segment 3 of the liver, which had the same features of vascularity as the ventral part of the primary liver tumor, and the second lumbar vertebra. Therefore, chemotherapy with gemcitabine and S-1 was started. Furthermore, three months later, some metastatic lymph nodes were confirmed around the head of the pancreas. Thereafter, although the lymph nodes were gradually increasing, new lesions did not appear, and the sizes of both the liver and bone metastases were stable. One year and four months after the first operation, we performed a resection of segment 3 of the liver and dissection of the lymph nodes to reduce the volume of the cancerous masses because we judged the chemotherapy to be effective.

The histological findings of the liver tumor were similar to those of the CoCC confirmed at the first operation (Fig. 6a, b), and an ICC component was not found in the tumor. All of the malignant lymph nodes were confirmed to metastasize from ICC (Fig. 6c, d).

Although recurrent lymph nodes appeared in the paraaortic region after the second operation, chemotherapy with gemcitabine and S-1 maintained stable disease. The patient survived with good quality of life for three years and five months after the first operation.

\section{Discussion}

$\mathrm{CoCC}$, which was first described by Steiner and Higginson $[1,2]$, is derived from the canals of Hering, where the 

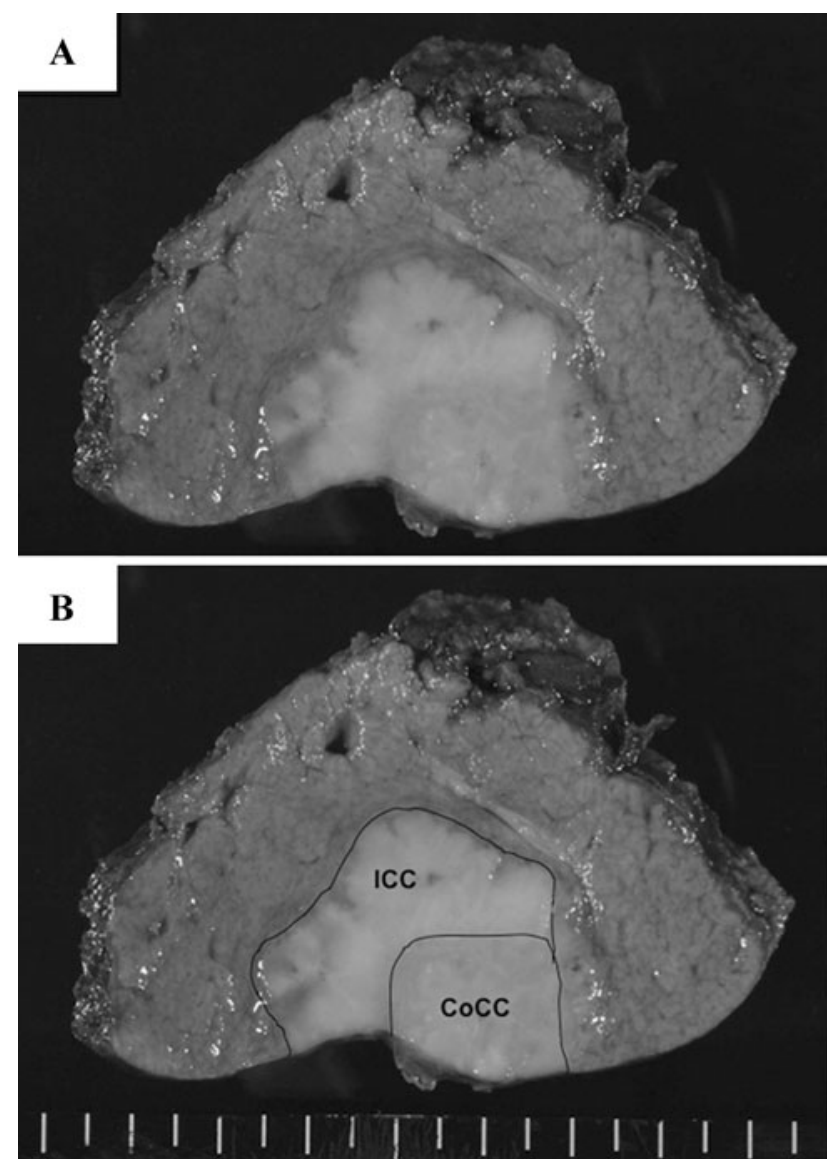

Fig. 3 The macroscopic appearance of the resected liver at the first operation. The tumor was composed of two portions (a). The portion of CoCC was surrounded by that of ICC, which was confirmed by histopathological examination (b)

hepatic progenitor cells are located, and is thought to have the junctional potential to differentiate into either $\mathrm{HCC}$ or ICC. CoCC is an extremely rare primary malignant tumor in the liver, with a frequency as low as $0.56 \%$ in Japan [3], so its clinical features have not yet been clarified.

Although there are only a few reports on the imaging features of $\mathrm{CoCC}$ [4-6], it is characterized as being peripherally located within the liver, with a lack of necrosis, calcification, and a fibrous capsule. In the dynamic, contrast-enhanced CT study, CoCC exhibited complete early enhancement with delayed washout or peripheral early ring-like enhancement with delayed centripetal filling. It is important to note that $\mathrm{CoCC}$ may be followed up as an atypical hemangioma, e.g., sclerosing hemangioma [7].

Immunohistochemical analysis of CoCC shows tumor cells with strong cytoplasmic positivity for CK7 and CK19, which are expressed in the bile duct epithelium, and positive staining for neural cell adhesion molecule
(NCAM); however, these tumor cells are negative for hepatocyte paraffin (Hep Par) 1 and CD10 expression [8]. Nakano regarded the dyeing pattern of EMA as the most important feature for the diagnosis of CoCC [9]. It is useful for differential diagnosis that the membrane on the luminal side is strongly positive for EMA in CoCC, while the cytoplasm is homogeneously positive in ICC.

The three most interesting points of this case are as follows. First, part of the CoCC transformed to ICC, which then increased rapidly, as shown by the changes in the CT findings over time. The characteristics of ICC in CT during both the early and delayed phases are usually thin, incomplete rim enhancements [10]. The central part of the tumor does not enhance during these phases; however, there may be prolonged enhancement in delayed-phase CT. In the $\mathrm{CT}$ images in this case, very small low-density portions in the area of the $\mathrm{CoCC}$, which was strongly enhanced in the early phase, increased with time and exhibited the abovementioned pictorial characteristic. Although CoCC is thought to have the potential to differentiate into either HCC or ICC, no report has clarified this process, although there have been some reports that they coexisted at the time of diagnosis [11] or that the CoCC image findings changed during progression [12].

The second point is the difference in potential malignancy between CoCC and ICC. Over a three-year observation period, although the CoCC increased slightly, the ICC increased rapidly once it was recognized by CT. This result may indicate that ICC is more malignant than CoCC in its ability to increase. The prognosis of patients suffering from $\mathrm{CoCC}$ is not clear due to the low incidence of CoCC. In one report [8], 10 of 29 patients showed intrahepatic recurrence $(n=7)$ or distant metastases $(n=3)$ after a mean 640-day disease-free survival, and five patients died from cancer-related diseases. In another report in Japan [9], 3 of 9 patients died 33 months, 14 months (HCC combined), and 1 month (HCC and ICC combined) after surgery.

The third point is the difference in the metastasis of CoCC and ICC. In our case, it was confirmed that all lymph node metastases were from ICC and the intrahepatic recurrence was composed of only CoCC. Komuta et al. [8] reported that intrahepatic metastases occurred in seven cases, lung metastases in two cases, and bone metastases in one case; however, whether the metastatic lesions were CoCC or another tumor type was not determined.

This case provided us with an interesting suggestion for the differentiation process from CoCC to ICC as well as for recurrence and treatment. We hope that the clinical features of CoCC will be made clear and that appropriate treatment will be established as a result of the accumulation of many such cases in the future. 
Fig. 4 Microscopic findings in hematoxylin-eosin staining. a CoCC portion. Tumor cells with eosinophilic cytoplasm proliferate in tubular structures with abundant fibrous stroma. b ICC portion
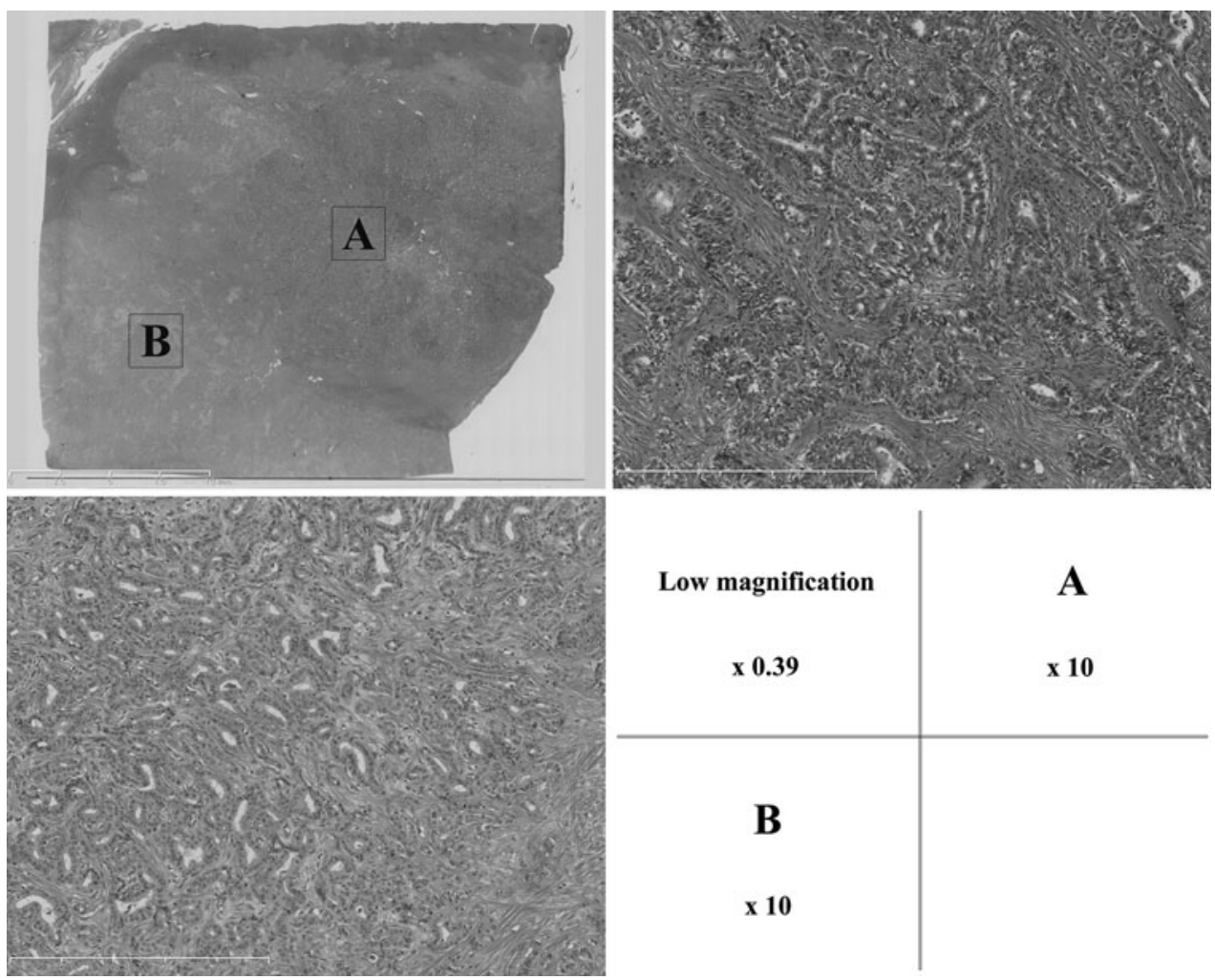

Fig. 5 Microscopic findings in EMA staining. a CoCC portion. The tumor cells strongly express EMA on the luminal surface. In contrast, tumor cells are positive for EMA in the cytoplasm in the ICC portion (b)
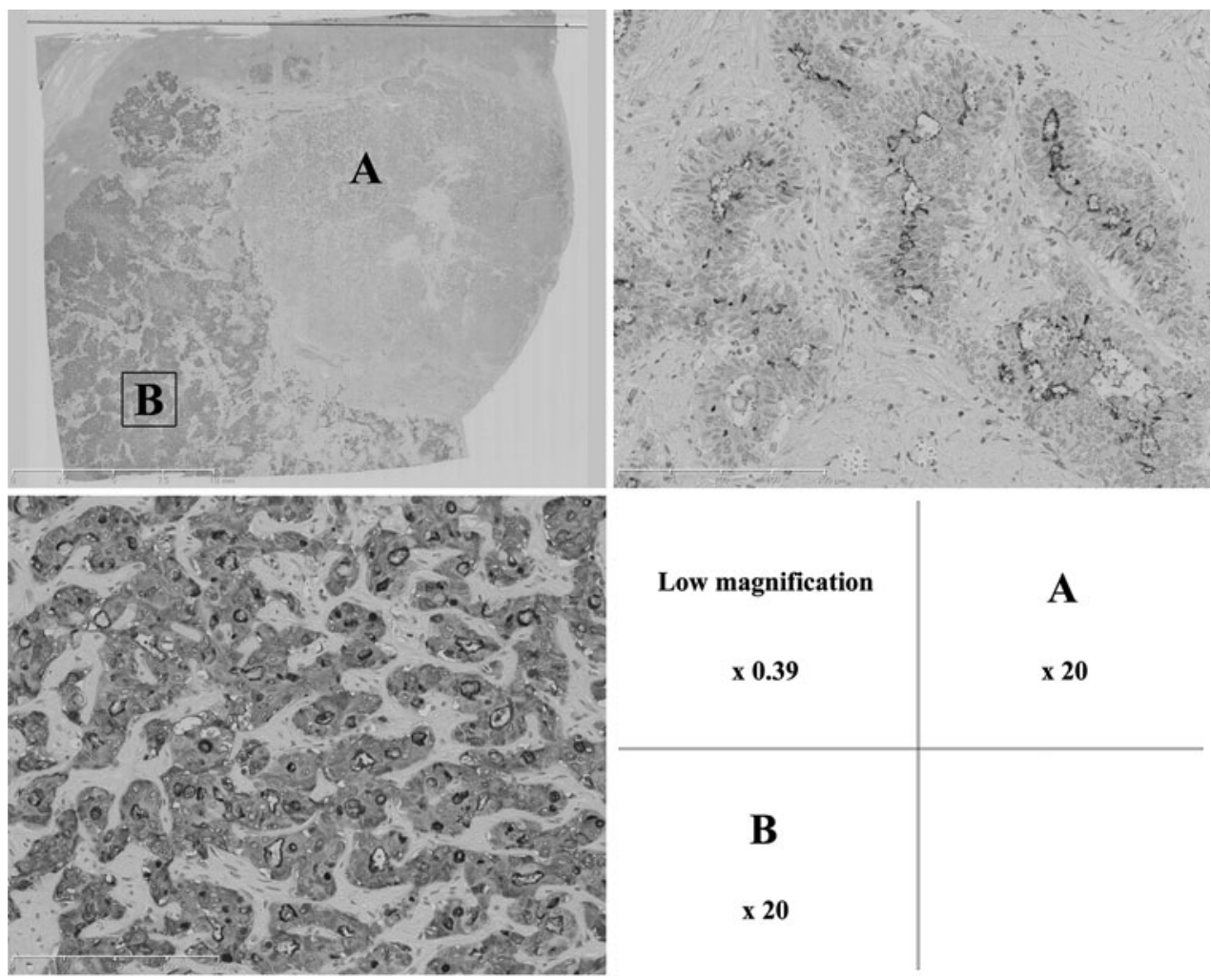
Fig. 6 Microscopic findings in EMA staining of the resected liver tumor $(\mathbf{a}, \mathbf{b})$ and metastatic lymph nodes $(\mathbf{c}, \mathbf{d})$ at the second operation
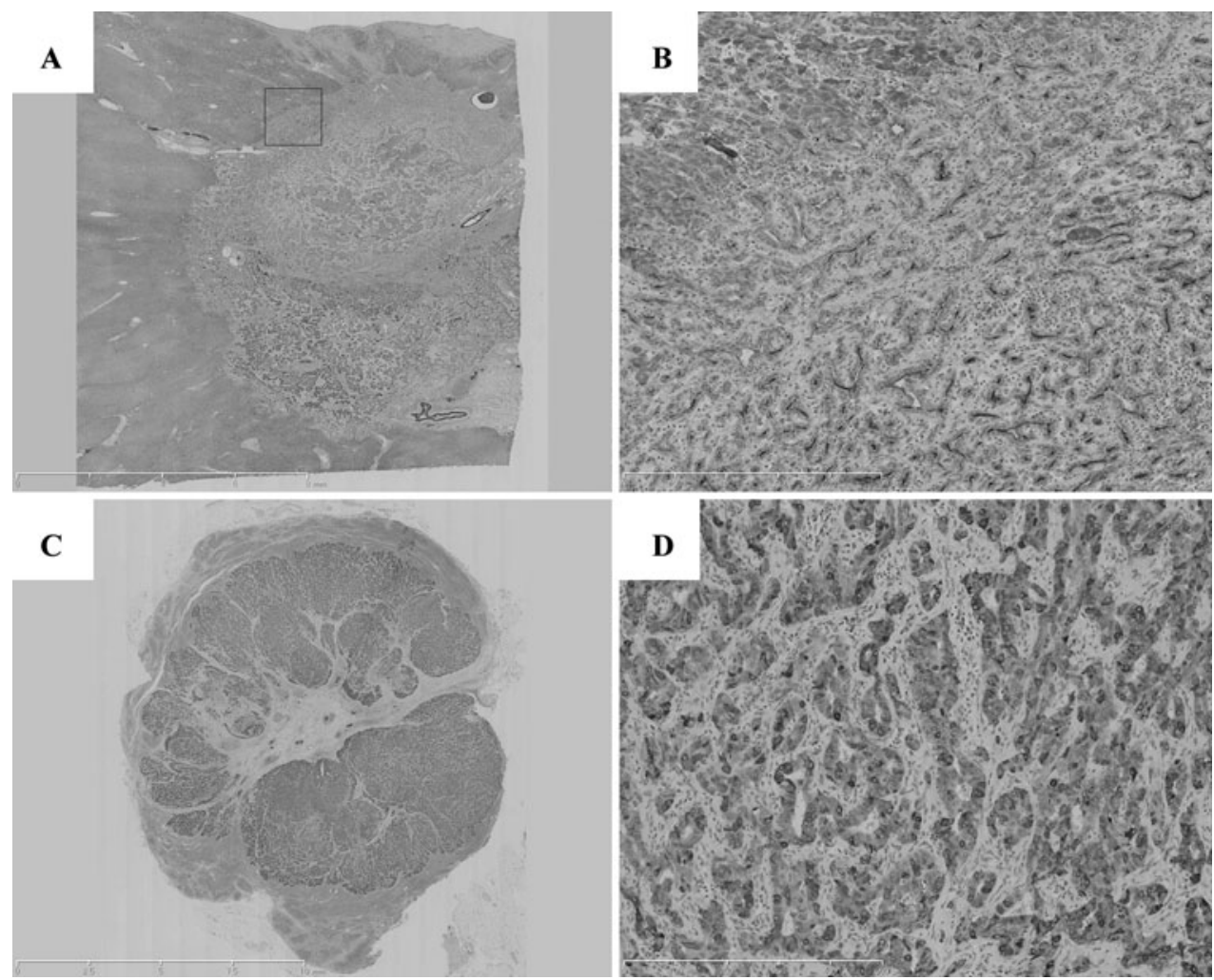

Conflict of interest The authors declare that they have no conflict of interest.

\section{References}

1. Steiner PE (1957) Carcinoma of the liver in the United States. Acta Unio Int Contra Cancrum 13:628-645

2. Steiner PE, Higginson J (1959) Cholangiolocellular carcinoma on the liver. Cancer 12:753-759

3. Shiota K, Taguchi J, Nakashima O et al (2001) Clinicopathologic study on cholangiolocellular carcinoma. Oncol Rep 8:263-268

4. Asayama Y, Tajima T, Okamoto D et al (2010) Imaging of cholangiolocellular carcinoma of the liver. Eur J Radiol 75:e120e125

5. Motosugi U, Ichikawa T, Nakajima $\mathrm{H}$ et al (2009) Cholangiolocellular carcinoma of the liver: imaging findings. J Comput Assist Tomogr 33:682-688

6. Fukukura Y, Hamanoue M, Fujiyoshi F et al (2000) Cholangiolocellular carcinoma of the liver: CT and MRI findings. J Comput Assist Tomogr 24:809-812
7. Koga $Y$, Nagahama H, Tateyama M et al (2012) A case of cholangiolocellular carcinoma combined with intrahepatic cholangiocarcinoma diagnosed after 4 years follow-up for hepatic hemangioma. Nihon Shokakibyo Gakkai Zasshi 109:231-239 (in Japanese)

8. Komuta M, Spee B, Borght SV et al (2008) Clinicopathological study on cholangiolocellular carcinoma suggesting hepatic progenitor cell origin. Hepatology 47:1544-1556

9. Han JK, Choi BI, Kim AY et al (2002) Cholangiocarcinomapictorial essay of $\mathrm{CT}$ and cholangiographic findings. Radiographics 22:173-187

10. Kanamoto M, Yoshizumi T, Ikegami T et al (2008) Cholangiolocellular carcinoma containing hepatocellular carcinoma and cholangiocellular carcinoma, extremely rare tumor of the liver: a case report. J Med Invest 55:161-165

11. Nakano M (2004) Pathology of cholangiolocellular carcinoma. Tan to Sui 25:343-349 (in Japanese)

12. Izawa N, Matsunaga K, Nagase $Y$ et al (2008) A case of cholangiolocellular carcinoma of which image findings changed during the time of progression. Kanzo 49:430-439 (in Japanese) 